\title{
Genetic Influences on Psychological Well-Being: A Nationally Representative Twin Study
}

\author{
Despina Archontaki, Gary J. Lewis, and Timothy C. Bates \\ University of Edinburgh
}

\begin{abstract}
Objective: Psychological well-being, or eudaimonia, features strongly in theories of human development and thriving. However, the factors of eudaimonia are debated, and their genetic architecture has not been studied in detail.

Method: A classical twin design was used to decompose behavioral variance into genetic and environmental components implemented in a multigroup, multivariate structural equation modeling framework. Subjects were 837 pairs of adult U.S. twins from the nationally representative MIDUS II sample. Psychological well-being was measured using the 42-item Ryff Psychological Well-Being Scale, which assesses autonomy, environmental mastery, personal growth, positive relations with others, purpose in life, and self-acceptance.

Results: Substantial genetic influences were observed on all components of well-being. Attempts to model these six factors as reflecting a single common psychological mechanism gave a poor fit to the data. The best-fitting model supported the existence of five distinct genetic effects. Effects of shared environment were weak and nonsignificant. Unique environmental effects for all measures were mostly trait specific.

Conclusions: These results indicate that psychological well-being is underpinned by a general genetic factor influencing self-control, and four underlying biological mechanisms enabling the psychological capabilities of purpose, agency, growth, and positive social relations.
\end{abstract}

Keywords: psychological well-being, well-being, eudaimonia, self-control, twin study, genetics

\section{Introduction}

Eudaimonic well-being (reflected in traits of environmental mastery, personal growth, purpose in life, self-acceptance, autonomy, and positive relations with others) is increasingly recognized as an important domain of individual differences (Ryff, 1989). However, the heritability of eudaimonic wellbeing has received little attention to date, and its fine-grained structure is unclear. Here we examine these issues in a representative twin sample using a broad-bandwidth assessment of eudaimonic well-being .

The concept of eudaimonia has its origins in Aristotelian thought regarding the nature of a good life. Aristotle defined this good life as the end result of " $a$ virtuous activity of soul of a certain kind" (Aristotle, 1925/1998, p. 18) and described the path to eudaimonia as a conscious and lifelong active exercise of intellect and character virtues. Current models of eudaimonia draw on theories of human development, self-actualization, and the means of its accomplishment, such as those of Erikson, Allport, and Maslow (Ryff, 1989). Based on these ideas, several authors have operationalized the construct (Waterman, 1993). Perhaps the most widely used measures are the sixfactor Scales of Psychological Well-Being (Ryff, 1989) assess- ing autonomy, personal growth, self-acceptance, purpose in life, environmental mastery, and positive relations with others (note that measures deigned to reflect eudaimonia typically refer to this construct as "psychological well-being," and we use this term when referring to these scales). The Ryff psychological well-being model captures the Aristotelian emphasis on the qualities of belonging and benefiting others, flourishing, thriving, and exercising excellence, although it omits some intellect qualities, character traits, and values that Aristotle would have emphasized, for instance, wisdom, bravery, generosity, and justice (Aristotle, 1925/1998).

\section{Structure of Eudaimonia}

Several theories have been proposed regarding the structure of eudaimonic well-being. Ryan and Deci (2001) suggest that psychological well-being is underpinned by three basic psy-

Correspondence concerning this article should be addressed to Timothy $\mathrm{C}$. Bates, Centre for Cognitive Ageing and Cognitive Epidemiology, University of Edinburgh, 7 George Square, Edinburgh EH8 9JZ, United Kingdom. Email: tim.bates@ed.ac.uk. 
chological needs: autonomy, competence, and relatedness. By contrast, Ryff (1989) has proposed a six-factor model, whereas other researchers have suggested that the components of eudaimonic well-being overlap so highly that they form a single construct (Springer, Hauser, \& Freese, 2006). At a phenotypic level, analyses support the existence of a general factor related to all components of psychological well-being, as well as additional factors distinguishing the subscales (e.g., Abbott et al., 2006).

One approach to articulating the structure of psychological traits is the use of genetically informed designs, such as the twin study, as used here. Considerable research has already been focused on genetic bases of hedonic or subjective wellbeing. While much of this has been univariate (Lykken \& Tellegen, 1996; Nes, Roysamb, Tambs, Harris, \& ReichbornKjennerud, 2006; Roysamb, Tambs, Reichborn-Kjennerud, Neale, \& Harris, 2003), multivariate approaches have begun to reveal links between subjective well-being and personality (Weiss, Bates, \& Luciano, 2008), and also relationships among different measures of subjective well-being (Bartels \& Boomsma, 2009). In this latter study, four different measures of subjective well-being were assessed in over 2,000 families of twins. Multivariate genetic modeling indicated that the four well-being measures reflected common underlying additive and nonadditive general genetic factors, with two additional specific genetic factors accounting for heritable differences among the four traits.

One study has recently examined the genetic structure of eudaimonia (Keyes, Myers, \& Kendler, 2010). These authors used a brief measure of psychological well-being (Ryff \& Keyes, 1995). As Bartels and Boomsma (2009) reported for subjective well-being, this analysis highlighted evidence for a common mechanism mediating genetic and environmental variance not only for eudaimonic well-being, but also for hedonic and social well-being (Keyes et al., 2010). However, with limited coverage of eudaimonia afforded by brief scales, the genetic structure of eudaimonia itself remains underexplored. Here we sought specifically to investigate the structure of the genetic and environmental influences on eudaimonic traits utilizing a broader-bandwidth scale (42-item, six-scale measure of eudaimonic well-being) developed for the second wave of testing in the MacArthur Foundation Survey for Midlife Development in the United States (MIDUS II) National Study of Health and Well-Being (Ryff et al., 2007).

\section{Hypotheses and Aims}

Following previous research (Keyes et al., 2010), we predicted that the six components of eudaimonia would share common genetic influences. Strong versions of this hypothesis were, first, that this shared variance would reflect a single psychological mechanism mediating all genetic and environmental effects (Hypothesis 1: common pathway model) or, in a less restrictive model, that while genes and environment effects might work through different pathways, all the covariance among eudaimonia measures would be mediated by general factors (Hypothesis 2: independent pathway model).

With the extra fidelity and bandwidth afforded by a longer and more comprehensive measure of eudaimonia, our preferred hypothesis predicted that a well-fitting model would reflect not only general influences, but also evidence for multiple significant additional genetic mechanisms underlying each of the components of well-being (Hypothesis 3).

\section{Design and Analysis}

A classical twin design was used comparing the resemblances of identical (monozygotic: MZ) and fraternal (dizygotic: DZ) twins. Structural equation modeling can be used to decompose the variance in traits into additive genetic (A), shared environment (C) and unique environmental (E) components. The covariance due to any additive genetic effects is set to 1 in the MZ twins, reflecting their $\sim 100 \%$ shared genome, and to $1 / 2$ in DZ twins, reflecting the fact that these twins, on average, share half their variable genetic inheritance. Shared environment covariance between Twin 1 and Twin 2 in each group is set to 1 , and covariance due to unique effects is set to zero in both groups, reflecting influences that make siblings different from each other.

In testing our hypotheses regarding the structure of psychological well-being, we used a saturated multivariate Cholesky decomposition of additive genetic, shared environmental, and unique environmental covariance between the measures as a base model. The Cholesky specifies as many factors for each of $\mathrm{A}, \mathrm{C}$, and $\mathrm{E}$ components as there are variables (sources of variance), which are arranged in a lower-triangular format so each factor has one fewer loading than that of the preceding factor (see Figure 1).

A range of substantive theoretical claims can be tested within the behavior genetic framework (Boomsma, Busjahn, \& Peltonen, 2002). Two in particular are used here: the common pathway and independent pathway models. The common pathway model (see Figure 2) provides a sophisticated test of the theoretical claim that covariance among measures reflects a single or common psychological mechanism. This common mechanism is instantiated as a latent variable through which all shared genetic and environmental influences are mediated (Kendler, Heath, Martin, \& Eaves, 1987). As can be seen in Figure 2, the model also allows for specific A, C, and E effects on each variable, in addition to the shared influence from the common trait. Mechanistically, this common trait would often reflect a common brain system through which genetic, family, social, and physical influences all gain their influence on the behaviors, and this has been found to be a valid representation of at least some complex psychological traits (cf. Lewis \& Bates, 2010).

A less restrictive model retains the general factors of the common pathway model but drops the requirement that genes and environment are expressed via a central mental mechanism, instead positing separate general factors for A, C, and E. 


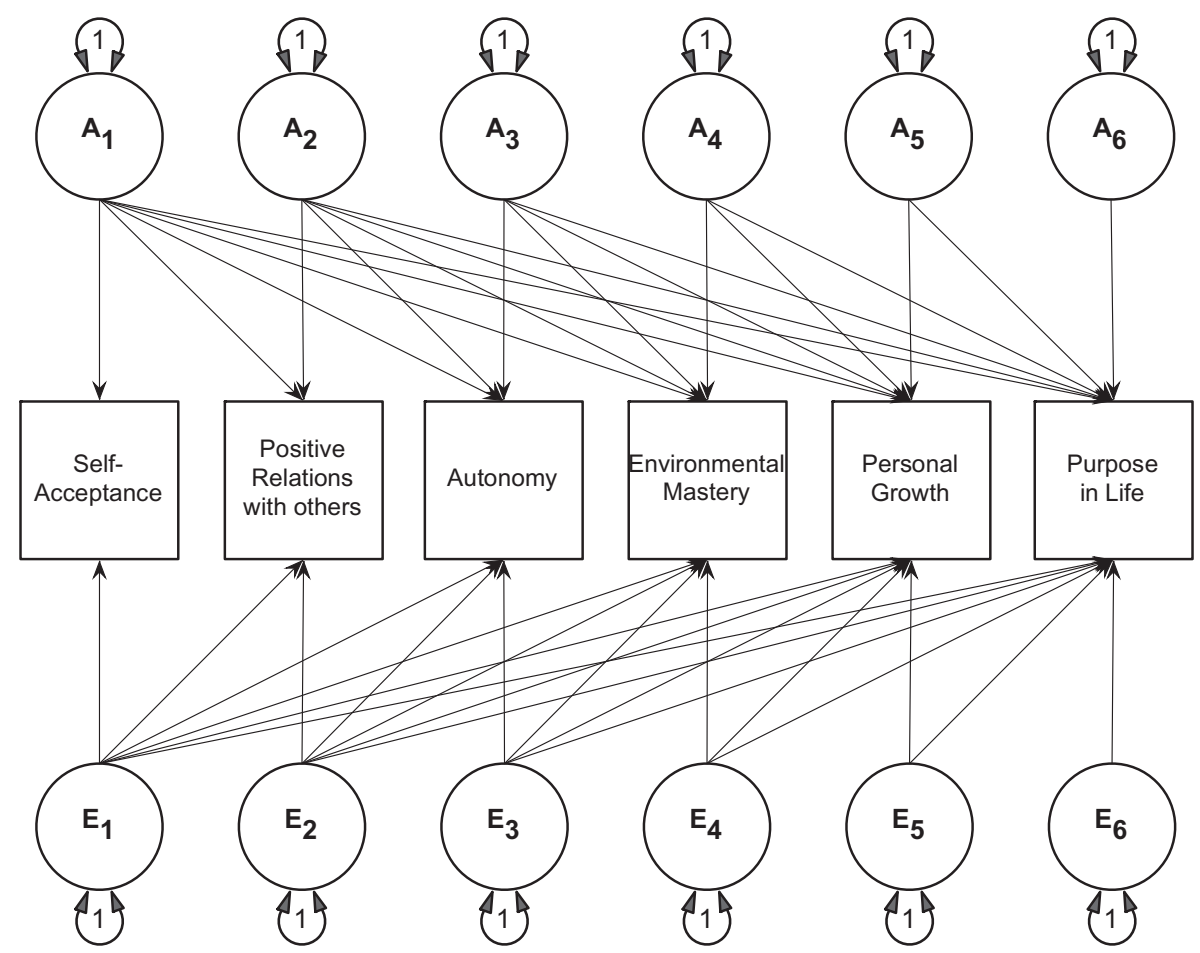

Figure I Saturated baseline model:The Cholesky decomposition.Additive genetic $\left(A_{n}\right)$ and unique environmental $\left(E_{n}\right)$ latent factors are shown here. Common environment (or dominance) effects are also included in the saturated base model (Model 0 ).

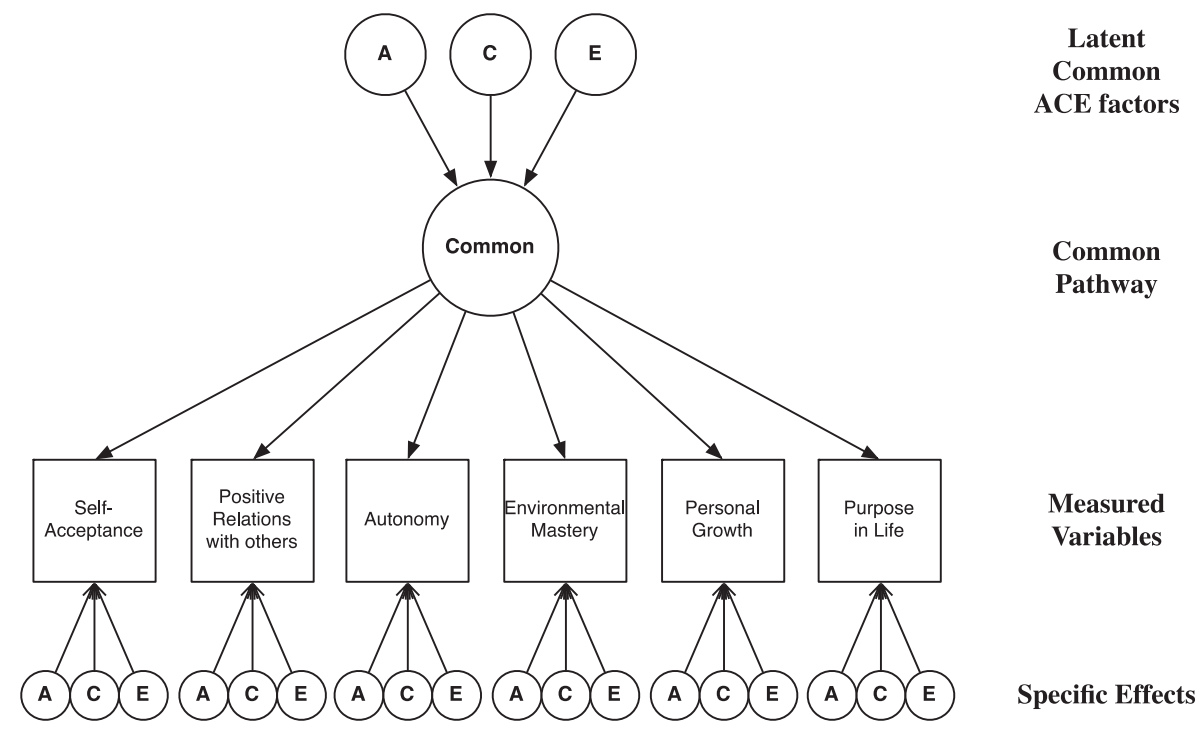

Figure 2 Common pathway model: All general effects operate through a common pathway, with specific effects operating on single traits. A, C, and E are additive genetic, common environmental, and unique environmental latent factors, repsectively.

This model, termed the independent pathway model (see Figure 3), requires that the general factor or covariance among all the traits reflects the influence of single sets of genes and environments with common influences, but it allows these to work independently of each other, rather than through their effect on a common mechanism.
The fit of these theoretical models can be explicitly tested by comparing their fit with that of the saturated baseline model (in this case, the Cholesky shown schematically in Figure 1) and with subsequent nested models (Neale \& Maes, 1996). Whether a reduced model (i.e., one with fewer parameters) is preferred is evaluated using measures of information criteria, 


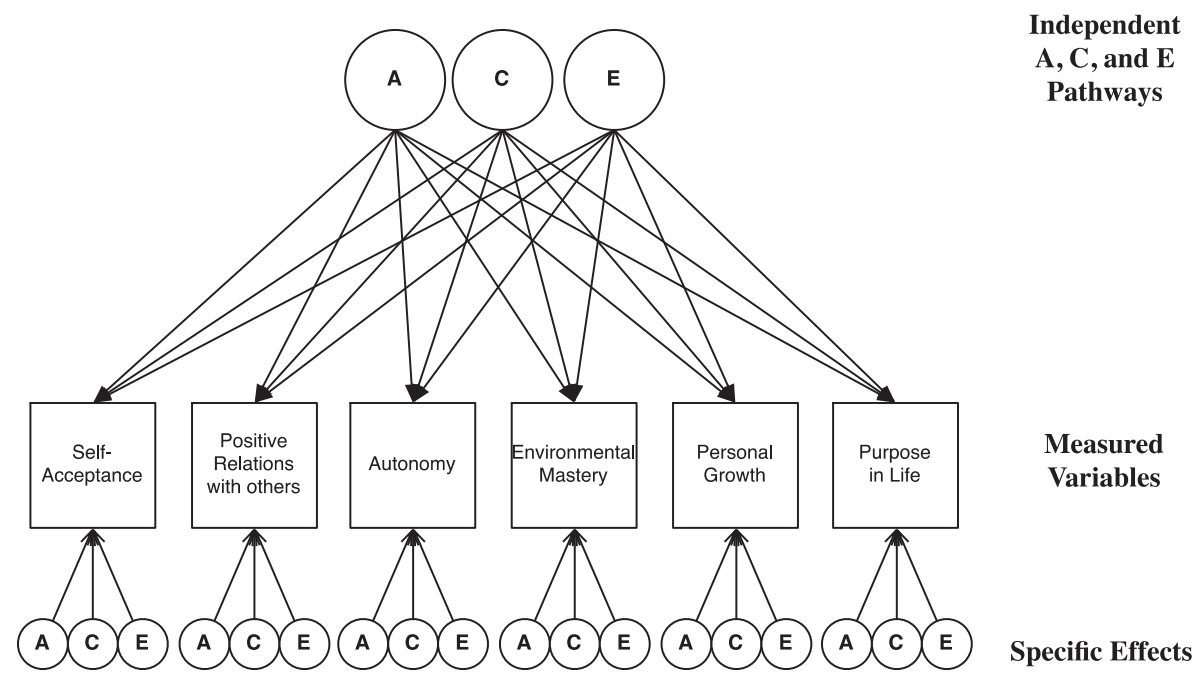

Figure 3 Independent pathway model. A, C, and E are additive genetic, common environmental, and unique environmental latent factors, respectively.

such as the Akaike information criterion (AIC; Akaike, 1974), and by a chi-square ratio test on difference in the $-2 \times \log$ likelihood, with degrees of freedom specified by the change in number of free parameters.

Prior to analyses, the effects of age and sex were residualized as these influences can inflate twin-pair correlations and confound cohort effects present in samples with large age differences, as is the case in the present sample (McGue \& Bouchard, 1984). All subsequent analyses were conducted using standardized residuals. All models were estimated using raw data and maximum likelihood methods and multigroup structural models implemented in OpenMx (Boker et al., 2011) under R (R Development Core Team, 2011). For the purposes of model comparison, all models were compared to a saturated baseline model (the Cholesky decomposition; Neale \& Cardon, 1992).

Hypotheses 1 and 2 proposed common pathway and independent pathway models to account for variance in psychological well-being. Hypothesis 3 predicted that covariance among the six psychological well-being traits could not be reduced to a single shared pathway and residuals, but that psychological well-being reflects multiple genetic influences distinguishing the six specific scales.

\section{METHOD}

\section{Sample}

Subjects were drawn from the MacArthur Foundation Survey for MIDUS, a nationally representative sample of 50,000 households selected by random telephone dialing process (Brim, Ryff, \& Kessler, 2004). Zygosity was ascertained using DNA microsatellite markers extracted from buccal swab samples. The study sample comprised $240 \mathrm{MZ}$ pairs (mean age $=44.0$ years, $S D=11.9$ ), $357 \mathrm{DZ}$ same-sex pairs (mean age $=45.2$ years, $S D=12.5)$ and 240 opposite-sex DZ pairs (mean age $=45.9$ years, $S D=12.0$ ).

\section{Measures}

Ryff's Scales of Psychological Well-Being. Eudaimonic well-being was assessed using the 42-item Scales of Psychological Well-Being (Ryff et al., 2007). The six scales (and an example item for each scale) are as follows: Autonomy, "I tend to be influenced by people with strong opinions"; Environmental Mastery, "I am quite good at managing the many responsibilities of my daily life"; Personal Growth: "I think it is important to have new experiences that challenge how you think about yourself and the world"; Positive Relations With Others: "People would describe me as a giving person, willing to share my time with others"; Purpose in Life, "Some people wander aimlessly through life, but I am not one of them"; and Self-Acceptance: "In many ways I feel disappointed about my achievements in life" (reverse scored). All responses were made on 7-point scales ranging from 1 (strongly agree) to 7 (strongly disagree). Scale scores were computed as the sum of relevant items, reversing items where appropriate. These measures have adequate reliability ( $\alpha=.70-.84$; Ryff et al., 2007).

\section{RESULTS}

Descriptive statistics for the Scales of Psychological WellBeing are provided, separately for each sex, in Table 1 . The results for each sex were highly similar in both means and variances, and univariate modeling of sex limitation revealed no significant effects. All data used in genetic modeling were residualized for sex.

The phenotypic (observed) structure of the six scales is given in Table 2, using one twin from each pair to avoid inflat- 
Table I Descriptive Statistics for Psychological Well-Being Scales

\begin{tabular}{|c|c|c|c|c|c|c|c|}
\hline Scale & Total N & Mean & $S D$ & Mean & $S D$ & Mean & $S D$ \\
\hline Purpose in Life & $\mathrm{I}, 745$ & 16.47 & 3.66 & 16.94 & 3.42 & 16.67 & 3.56 \\
\hline Positive Social Relations & $\mathrm{I}, 745$ & 16.76 & 3.99 & 15.78 & 4.07 & 16.33 & 4.05 \\
\hline Personal Growth & $\mathrm{I}, 744$ & 18.07 & 3.03 & 17.89 & 2.96 & 17.99 & 3.00 \\
\hline
\end{tabular}

Table 2 Phenotypic Correlations (Using One Twin From Each Pair)

\begin{tabular}{lcccc}
\hline Scale & Acceptance & Purpose in Life & Positive Relations & Personal Growth \\
\hline Purpose in Life & 0.29 & & & Autonomy \\
Positive Social Relations & 0.53 & 0.37 & 0.41 & 0.26 \\
Personal Growth & 0.42 & 0.39 & 0.41 & 0.24 \\
Autonomy & 0.35 & 0.23 & 0.40 \\
Environmental Mastery & 0.62 & & 0.36 \\
\hline
\end{tabular}

Table 3 Twin Correlations, Separately by Zygosity

\begin{tabular}{ccccccr}
\hline & Acceptance & Purpose in Life & Positive Relations & Personal Growth & Autonomy & Mastery \\
\hline MZ & 0.47 & 0.30 & 0.38 & 0.38 & 0.41 & 0.35 \\
DZ & 0.14 & 0.15 & 0.12 & 0.22 & 0.04 & 0.10 \\
\hline
\end{tabular}

Note. $M Z$ = monozygotic; $\mathrm{DZ}$ = dizygotic.

Table 4 Mean (TI-T2 T2-TI) Cross-Twin Cross-Trait Correlations

\begin{tabular}{|c|c|c|c|c|c|c|}
\hline Acceptance & - & 0.12 & 0.32 & 0.26 & 0.22 & 0.32 \\
\hline Purpose & 0.12 & - & 0.21 & 0.26 & 0.14 & 0.17 \\
\hline Growth & 0.05 & 0.09 & 0.06 & - & 0.23 & 0.27 \\
\hline Autonomy & 0.08 & 0.01 & 0.10 & 0.12 & - & 0.26 \\
\hline Mastery & 0.13 & 0.09 & 0.12 & 0.13 & 0.04 & - \\
\hline
\end{tabular}

Note. Monozygotic twins in upper triangle; dizygotic twins in lower triangle. TI = twin I; T2 = twin 2.

ing correlations due to familial covariance. Table 3 shows the twin correlations for each trait, separately for the two zygosities. As can be seen, the MZ correlations in all cases substantially exceeded those of the DZ pairs. In most cases, MZ correlations were over double the DZ correlations. This is suggestive of dominance. As the classical twin design cannot model both $\mathrm{C}$ and $\mathrm{D}$ (dominance) effects simultaneously, we compared ACE and ADE models. These were not substantively different (difference in $-2 \times \log$ likelihood $=15.2, \quad \mathrm{AIC}=$ $31,733.58$ vs. $31,733.58)$, and, importantly, both shared environment and dominance effects could be dropped without significant loss of fit $\left(\mathrm{AIC}=31,711.46, \chi^{2}(21)=19.88\right.$, $p=.53)$.
Table 4 gives the mean cross-twin cross-trait correlations. Differences in cross-twin cross-trait correlations between the two zygosities are indicative of the sources of covariance between the different traits. It is apparent here that there is support for significant genetically based covariance linking the traits and, with a positive manifold of relations, a significant genetic general factor of some form. We next proceeded to the theoretically motivated analysis of models of eudaimonic well-being.

Statistical information on this baseline model (Model 0) and the subsequent theoretical comparison models are shown in Table 5. The first theoretical model tested Hypothesis 1. As noted above, the common pathway model provides a sophisti- 
cated test of whether a general psychological mechanism can account for the observed data by implementing a latent variable through which all shared genetic and environmental influences are mediated (Kendler et al., 1987). As might be expected from the low phenotypic correlations between the traits, this model fit poorly in comparison to the baseline (Model 0; $\Delta \chi^{2}(37)=215.27, p<.001 ;$ AIC $=31,890.05$ vs. 31,748.78; see Table 5, Model 1).

We next tested Hypothesis 2: This hypothesis specified that a general factor fits the data, but that genetic and environmental covariance between the eudaimonic traits reflects independent genetic and environmental pathways. This model (Model 2 ), however, also fit poorly, $\Delta \chi^{2}(27)=92.52, p<.001$ ) (see Table 5).

With both of the restrictive general factor models fitting poorly, we proceeded to examine more complex genetic structures, utilizing both general and group genetic factors to explain the data (Hypothesis 3: the preferred model). Using the Cholesky model as a platform for determining the fine-grained genetic structure of eudaimonia, we first tested whether the effects of shared environment were significant; these were observed to be small and could be dropped with negligible loss of fit (Model 3: $\Delta \chi^{2}(21)=3.64, p=.99$ ). We next examined whether further simplifications of genetic effects were possible.

To test how many genetic factors (distinct sources of genetic variance) are involved in psychological well-being, we fitted models attempting to drop one or more of the factors from the Cholesky. Dropping the sixth factor led to an improved fit by AIC criterion (AIC reduced from 31,710.42 to $31,708.42$ ), with virtually no change in $\log$ likelihood $\left(\Delta \chi^{2}(1)=<.001, p<.99\right)$. Attempting to drop the fifth factor, however, led to a worse fit by AIC and chi-square criteria $\left(31,711.41\right.$ vs. $\left.31,708.42 ; \Delta \chi^{2}(1)=6.98, p=.03\right)$, confirming that there are at least five distinct genetic influences on psychological well-being.

To better understand the nature of these genetics influences, the base Cholesky was modified. In the best-fitting model by
AIC criteria, nine additional non significant additive genetic paths could be removed. This final model of additive genetic effects is shown in Figure 4.

\section{Modeling the Unique Environment}

A benefit of the twin design is not only that genetic effects can be modeled, but also that the decomposition supports the modeling of environment effects - testing theories of the environmental origins of individual differences. Reductions in unique environment effects were also explored. As existing theories do not make claims about the environmental structure of eudaimonia, here we proposed and tested a series of hypotheses.

First, we tested whether unique environment effects showed any structure beyond trait-specific factors, perhaps reflecting measurement error. This model, setting all paths below the diagonal to zero, fit poorly, $\left.\Delta \chi^{2}(15)=365.7, p<.0001\right)$. Next, we tested whether the covariance among the traits could be accounted for by a single general factor and six trait-specific effects. This also fit very poorly, $\Delta \chi^{2}(10)=135.63, p<.0001$ ), suggesting the unique environment was reflecting more than simply noise and a general measurement effect. Finally, we tested whether the environmental structure mirrored that found in the genetic model, perhaps reflecting a common-path effect at the level of the group factors identified genetically. This model too, however, fit poorly, $\Delta \chi^{2}(5)=101.37, p=2.7 \times$ $\left.10^{-20}\right)$. There was, then, no obvious meaningful structure to the unique environment effects. In our final model, then, E was reduced by dropping paths that did not contribute significantly to model fit by AIC (see Figure 5 for unique environment path loadings and confidence intervals).

The final model contained five distinct genetic latent traits, no shared environment effects, and a unique environment structure dominated by specific loadings. The genetic (and unique environmental) correlations between the scales are shown in Table 6 and reflect the shared genetic influences and, typically, very low common influences of the unique environment.

Table 5 Parameters, Fit Statistics, and Comparison Statistics for All Models

\begin{tabular}{|c|c|c|c|c|c|c|c|}
\hline $\begin{array}{l}\text { Model } 0 \\
\text { (Saturated Cholesky) }\end{array}$ & NA & 69 & 52256.78 & 31748.78 & - & - & - \\
\hline Hypothesis I: common pathway & & & & & & & \\
\hline $\begin{array}{l}\text { Model } 2 \\
\text { Hypothesis 2: independent pathway }\end{array}$ & Model 0 & 42 & 52349.30 & 31787.30 & 92.52 & 27 & $<.001$ \\
\hline Reduced $A$ & Model 3 & 40 & 52267.67 & 31701.67 & II.26 & 9 & .51 \\
\hline $\begin{array}{l}\text { Final Model } \\
\text { Reduced A and E (see Figure I) }\end{array}$ & Reduced A & 35 & 52277.60 & 31701.60 & 9.92 & 5 & .08 \\
\hline
\end{tabular}

Note. $\mathrm{EP}=$ number of estimated parameters; $-2 \mathrm{LL}=$ minus $2 \times \log$-likelihood; $\mathrm{AIC}=$ Akaike Information Criterion; $\Delta-2 \mathrm{LL}=$ change in minus $2 \times \log$ likelihood; $\Delta d f=$ change in degrees of freedom; $p=p$-value (smaller values indicate worse fit). 


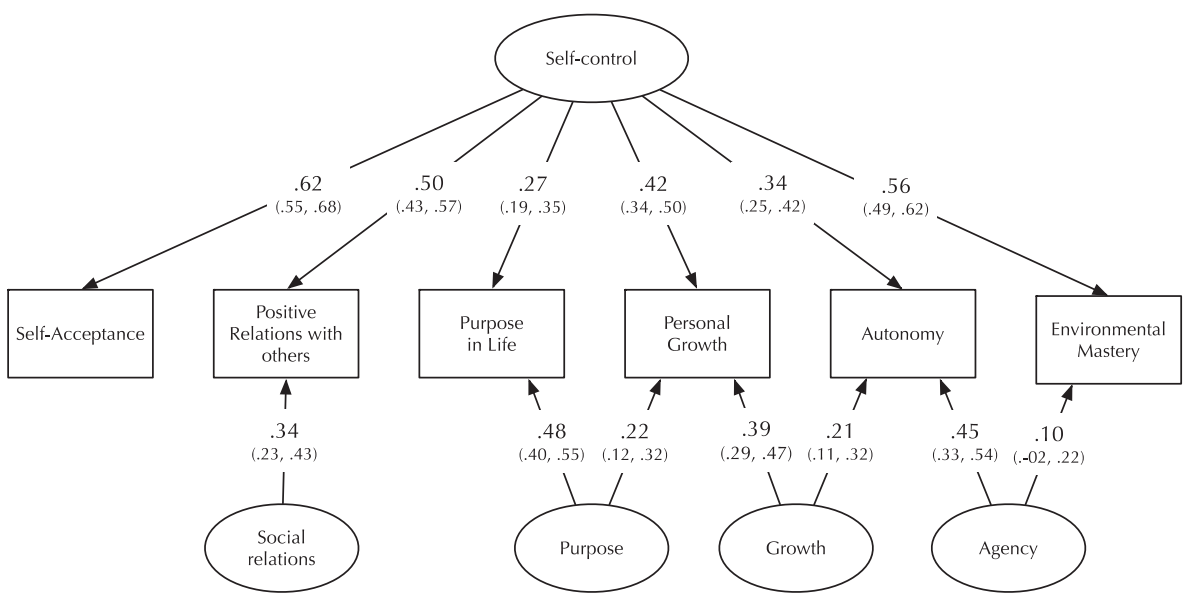

Figure 4 Final model of genetic influences on the facets of eudaimonia. Path values are path coefficients from the standardized solution (square these to get standardized variance components). Values in parentheses indicate $95 \%$ confidence intervals. All paths were significant, and no additional paths improved model fit significantly.

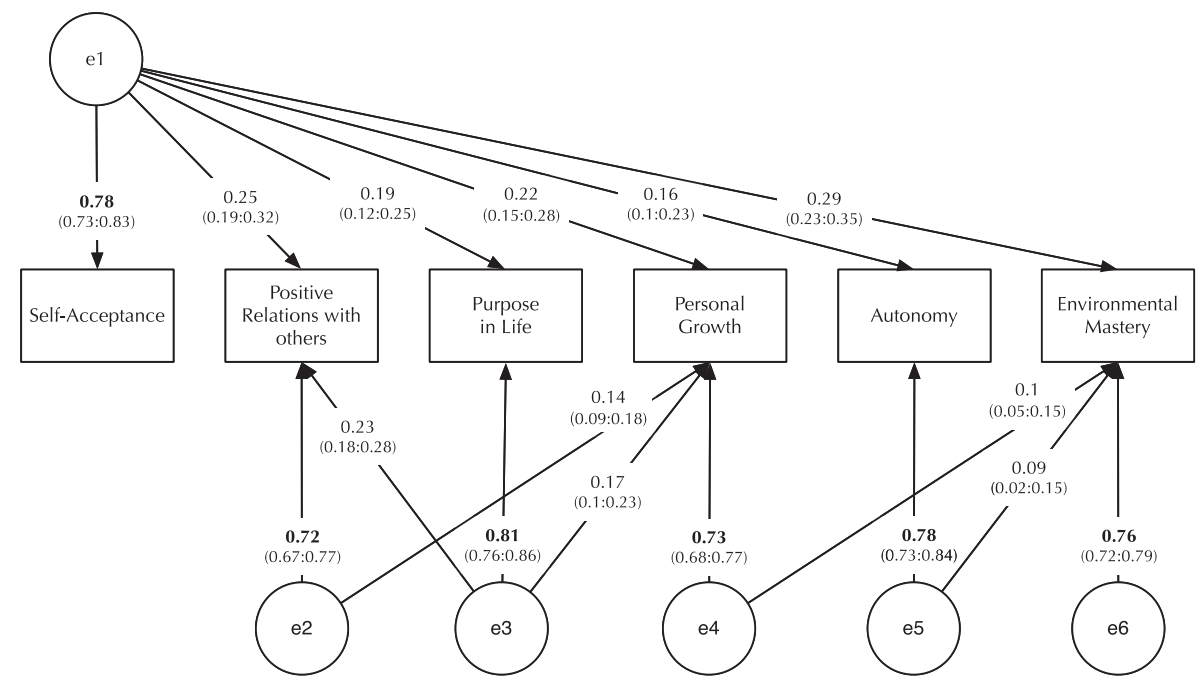

Figure 5 Final model of unique environment influences on the facets of eudaimonia. Path values are path coefficients from the standardized solution (square these to get standardized variance components). Values in parentheses indicate $95 \%$ confidence intervals. All paths were significant, and no additional paths improved model fit significantly.

Table 6 Table of Genetic Correlations

\begin{tabular}{lccccc}
\hline & Acceptance & Purpose & Relations & Growth & Autonomy \\
\hline Purpose & $0.49(.23)$ & & & & \\
Relations & $0.82(.32)$ & $0.40(.35)$ & & & \\
Growth & $0.68(.28)$ & $0.64(.27)$ & $0.56(.31)$ & & \\
Autonomy & $0.56(.21)$ & $0.27(.05)$ & $0.46(.07)$ & $0.61(.06)$ & \\
Mastery & $0.98(.35)$ & $0.48(.08)$ & $0.81(.11)$ & $0.67(.21)$ & $0.69(.17)$
\end{tabular}

Note. Unique environmental correlations are in parentheses.

A general additive genetic factor, which we termed selfcontrol, loaded on all six facets of psychological well-being, with its highest loading on self-acceptance (.62) and significant loadings on all other traits. The second genetic mechanism, which we termed purpose, loaded on two variables: purpose in life (.48) and personal growth (.22). The third genetic factor, which we termed social relations, loaded on positive relations with others (.34). A loading of this factor on autonomy, estimated at .10, was dropped as nonsignificant but may help interpret this genetic influence on social behavior. The fourth factor, which we named growth, loaded on personal growth (.39) and autonomy (.21), with a nonsignificant loading of .17 on environmental mastery. The final genetic factor was termed agency and loaded on autonomy (.45) and environmental mastery (.10).

\section{DISCUSSION}

The main aim of this article was to examine the genetic structure of eudaimonia within the framework of Ryff's (1989) 
six-factor theory of psychological well-being. The results indicated a significant genetic influence on all scales of psychological well-being, confirming our hypothesis that eudaimonia reflects substantial genetic influences. Furthermore, both a (substantial) general factor and four additional significant specific genetic factors were required to account for these heritable effects, suggesting the existence of a complex genetic structure containing both general and group-level influences, and revealing important biological commonalities between different facets of psychological well-being. No theoretically meaningful models of $\mathrm{E}$ fit well, with the final model consisting of large specific factors including error variance, and minor off-diagonal correlations. This finding, together with the lack of significant effects of $C$, suggests that environmental experiences are not, in general, the source of the psychological structure for well-being, supporting an argument previously made more generally by McCrae, Jang, Livesley, Riemann, and Angleitner (2001).

\section{General Factor of Eudaimonia}

Compatible with Keyes et al. (2010), we found evidence for a large general genetic factor, although in our analyses, this was insufficient to account for the genetic structure of the six facets of psychological well-being. In particular, a common pathway model, positing a general psychological mechanism through which both genetic and environmental effects on psychological well-being are mediated, fit poorly. This suggests that theoretical conceptions of well-being as a single mental system (Springer et al., 2006) do not capture this trait well. The lack of fit of the less restrictive independent pathway model further reduces the likelihood of one-dimensional interpretations of psychological well-being.

The general factor had its largest loading on selfacceptance, which in turn had no significant genetic loadings on any other factor. Self-acceptance is a central element of eudaimonia in Ryff's (1989) model, involving a sense of not having let one's self down expressed in a broad range of domains, including life story, personality, and achievements, and leading to confidence and positive attitudes toward the self. The current data confirm that self-acceptance is closely identified with the most general aspect of psychological wellbeing and suggest that differences in self-acceptance have a substantial genetic component.

This general biological mechanism must be able to exert control across all facets of eudaimonia, implying a top-down control mechanism over multiple systems processing emotional, reward-incentive, and motivational information related to psychological well-being. Because of this domain-general and top-down functionality, we provisionally labeled the factor "self-control." While many cortical regions are active during self-control activity, right ventrolateral prefrontal cortex activation (rVLPFC) is present regardless of domain (Cohen \& Lieberman, 2010). This suggests that rVLPFC is a candidate neural substrate for the general factor.

\section{Group Factors}

Turning to the four group factors, each of these additional sources of genetic variance in psychological well-being reflected loadings for individual scales on a subset of the facets of psychological well-being. Based on these loadings, we termed the four factors purpose, social relations, growth, and agency.

The purpose factor loaded significantly on both purpose in life and personal growth. Ryff (1989) described purpose in life as having intentions, goals, and a sense of direction. Personal growth, in turn, is linked to continued growth and the confronting of new challenges or tasks at different periods of life. Our analyses suggest that these two traits of seeking out new experiences and having a mindful sense of the future may be linked by common genetic origins. Ryff and Keyes (1995) have reported that both purpose in life and personal growth show similar declines with age, a finding compatible with a shared biology for the two traits. These two domains also map onto the performance and learning facets of goal orientation (Button, Mathieu, \& Zajac, 1996), suggesting that this factor may be related to, or underlie, differences in goal orientation.

Where the purpose factor indexed goal orientation, the fourth genetic factor, which we tentatively termed agency, was focused on the management of goals and having realized skills to achieve those goals. This factor loaded on both autonomy and environmental mastery, capturing the efficacy of the individual based on his or her self-understanding, evaluation and management of goals and responsibilities, and ongoing gain of skills. Data on interventions designed to promote autonomy also strengthen environmental mastery, supporting the linkage of these two factors (e.g., Altmaier, Russell, Kao, Lehmann, \& Weinstein, 1993). In terms of a biological substrate, it may be valuable to explore links of this factor to related concepts of willpower and ego in goal achievement (Baumeister, Bratslavsky, Muraven, \& Tice, 1998).

Finally, the social relations factor loaded on positive relations with others. Both developmental research (e.g., Birch \& Ladd, 1998) and research on attachment link effective social relations to underlying elevations of intimacy and trust and decreased evaluative cognitions about others (Hazan \& Shaver, 1987). Studies of both romantic and maternal attachment would locate this factor in a distributed system comprising activation of oxytocin- and vasopressin-rich reward systems and coupled deactivation of regions involved in evaluation (Bartels \& Zeki, 2004). Therefore, it may be worth examining links of this factor to these brain regions.

The analyses revealed several interesting factors regarding environmental effects. First, the relatively small and nonsignificant impact of shared environment effects is similar to that found for nongenetic family-level effects in other domains, including cognition (e.g., Deary, Spinath, \& Bates, 2006). Second, while the unique environmental variance could not be reduced simply to a general measurement effect and/or to random measurement error specific to each test, neither did it 
match the structure of a strong general factor and underlying group factors as identified genetically. Environments appear then to act outside the family level, and mainly on individual components of psychological well-being, or arbitrary pairs of these facets.

In summary, researchers have highlighted the need to understand the structure of eudaimonia and the number and nature of eudaimonic traits (Abbott et al., 2006; Ryff \& Keyes, 1995; Springer et al., 2006). The present results suggest possible answers to these questions: specifically, that psychological well-being may be reflect the functioning of a large general factor with biological origins, underpinned by four distinct biological systems reflecting additional genetic influences. Each of the six behavioral domains identified by the Ryff Scales of Psychological Well-Being (except Self-Acceptance, which reflected the general psychological well-being) was influenced by one or more of these systems. The results suggest that there is a specific biological architecture underlying traits of self-regulation suggested since antiquity, alongside intelligence, to be key to human capability.

\section{References}

Abbott, R. A., Ploubidis, G. B., Huppert, F. A., Kuh, D., Wadsworth, M. E., \& Croudace, T. J. (2006). Psychometric evaluation and predictive validity of Ryff's psychological well-being items in a UK birth cohort sample of women. Health Quality and Life Outcomes, 4, 76.

Akaike, H. (1974). A new look at the statistical model identification. IEEE Transactions on Automatic Control, 19, 716-723.

Altmaier, E. M., Russell, D. W., Kao, C. F., Lehmann, T. R., \& Weinstein, J. N. (1993). Role of self-efficacy in rehabilitation outcome among chronic low-back-pain patients. Journal of Counseling Psychology, 40, 335-339.

Aristotle. (1925/1998). The Nicomachean ethics (W. D. Ross, Trans.). Oxford: Oxford University Press.

Bartels, A., \& Zeki, S. (2004). The neural correlates of maternal and romantic love. Neuroimage, 21, 1155-1166.

Bartels, M., \& Boomsma, D. I. (2009). Born to be happy? The etiology of subjective well-being. Behavior Genetics, 39, 605-615.

Baumeister, R. F., Bratslavsky, E., Muraven, M., \& Tice, D. M. (1998). Ego depletion: Is the active self a limited resource? Journal of Personality and Social Psychology, 74, 1252-1265.

Birch, S. H., \& Ladd, G. W. (1998). Children's interpersonal behaviors and the teacher-child relationship. Developmental Psychology, 34, 934-946.

Boker, S., Neale, M., Maes, H., Wilde, M., Spiegel, M., Brick, T., et al. (2011). OpenMx: An open source extended structural equation modeling framework. Psychometrika, 76, 306-317.

Boomsma, D., Busjahn, A., \& Peltonen, L. (2002). Classical twin studies and beyond. Nature reviews: Genetics, 3, 872-882.

Brim, O. G., Ryff, C. D., \& Kessler, R. C. (Eds.). (2004). How healthy are we? A national study of well-being at midlife. Chicago: University of Chicago Press.
Button, S. B., Mathieu, J. E., \& Zajac, D. M. (1996). Goal orientation in organizational research: A conceptual and empirical foundation. Organizational Behavior and Human Decision Processes, 67, 26-48.

Cohen, J. R., \& Lieberman, M. D. (2010). The common neural basis of exerting self-control in multiple domains. In R. Hassin, K. Ochsner, \& Y. Trope (Eds.), Self-control in society, mind, and brain (pp. 141-162). Oxford: Oxford Scholarship Online Monographs.

Deary, I. J., Spinath, F. M., \& Bates, T. C. (2006). Genetics of intelligence. European Journal of Human Genetics, 14, 690-700.

Hazan, C., \& Shaver, P. (1987). Romantic love conceptualized as an attachment process. Journal of Personality and Social Psychology, 52, 511-524.

Kendler, K. S., Heath, A. C., Martin, N. G., \& Eaves, L. J. (1987). Symptoms of anxiety and symptoms of depression: Same genes, different environments? Archives of General Psychiatry, 44, 451457.

Keyes, C. L., Myers, J. M., \& Kendler, K. S. (2010). The structure of the genetic and environmental influences on mental well-being. American Journal of Public Health, 100, 2379-2384.

Lewis, G. J., \& Bates, T. C. (2010). Genetic evidence for multiple biological mechanisms underlying ingroup favoritism. Psychological Science, 21, 1623-1628.

Lykken, D., \& Tellegen, A. (1996). Happiness is a stochastic phenomenon. Psychological Science, 7, 186-189.

McCrae, R. R., Jang, K. L., Livesley, W. J., Riemann, R., \& Angleitner, A. (2001). Sources of structure: Genetic, environmental, and artifactual influences on the covariation of personality traits. Journal of Personality, 69, 511-535.

McGue, M., \& Bouchard, T. J., Jr. (1984). Adjustment of twin data for the effects of age and sex. Behavior Genetics, 14, 325-343.

Neale, M. C., \& Cardon, L. R. (1992). Methodology for genetic studies of twins and families (Vol. 67). Amsterdam: Kluwer Academic.

Neale, M. C., \& Maes, H. H. (1996). Methodology for genetics studies of twins and families (6th ed.). Dordrecht, The Netherlands: Kluwer.

Nes, R. B., Roysamb, E., Tambs, K., Harris, J. R., \& ReichbornKjennerud, T. (2006). Subjective well-being: Genetic and environmental contributions to stability and change. Psychological Medicine, 36, 1033-1042.

R Development Core Team. (2011). R: A language and environment for statistical computing (Version 2.12.X). Vienna, Austria: R Foundation for Statistical Computing. Retrieved from http:// www.R-project.org

Roysamb, E., Tambs, K., Reichborn-Kjennerud, T., Neale, M. C., \& Harris, J. R. (2003). Happiness and health: Environmental and genetic contributions to the relationship between subjective well-being, perceived health, and somatic illness. Journal of Personality and Social Psychology, 85, 1136-1146.

Ryan, R. M., \& Deci, E. L. (2001). On happiness and human potentials: A review of research on hedonic and eudaimonic well-being. Annual Review of Psychology, 52, 141-166.

Ryff, C. D. (1989). Happiness is everything, or is it? Explorations on the meaning of psychological well-being. Journal of Personality and Social Psychology, 57, 1069-1081. 
Ryff, C. D., Almeida, D. M., Ayanian, J. S., Carr, D. S., Cleary, P. D., Coe, C., et al. (2007). Midlife Development in the United States (MIDUS II), 2004-2006. Ann Harbor, MI: Inter-university Consortium for Political and Social Research (ICPSR).

Ryff, C. D., \& Keyes, C. L. M. (1995). The structure of psychological well-being revisited. Journal of Personality and Social Psychology, 69, 719-727.

Springer, K. W., Hauser, R. M., \& Freese, J. (2006). Bad news indeed for Ryff's six-factor model of well-being. Social Science Research, 35, 1120-1131.
Waterman, A. S. (1993). 2 Conceptions of happiness - Contrasts of personal expressiveness (eudaimonia) and hedonic enjoyment. Journal of Personality and Social Psychology, 64, 678691.

Weiss, A., Bates, T. C., \& Luciano, M. (2008). Happiness is a personal(ity) thing: The genetics of personality and well-being in a representative sample. Psychological Science, 19, 205210 . 